\section{Optic Neuropathy}

Kathleen L. Fuchs

Department of Neurology, University of Virginia Health System, Charlottesville, VA, USA

\section{Definition}

A change in or loss of vision as a result of damage to the optic nerve. For example, demyelination of the optic nerve (optic neuritis) is a common early symptom of multiple sclerosis (MS) that can result in temporary monocular blindness. Other causes of optic neuropathy include compression lesions (i.e., tumors), ischemia, Leber's hereditary optic neuropathy, and chronic relapsing inflammatory optic neuropathy (CRION), which is characterized by recurring episodes of optic neuritis marked by severe visual loss and orbital pain. In this condition, a contrast-enhanced MRI may show swelling of the optic nerve but no evidence of the demyelinating lesions in the brain characteristic of MS.

\section{Cross-References}

Multiple Sclerosis

- Optic Nerve

- Visual Evoked Potentials

\section{References and Reading}

Miller, D., \& Compston, A. (2006). The differential diagnosis of multiple sclerosis. In A. Compston et al. (Eds.), McAlpine's multiple sclerosis (4th ed., pp. 389-437). Philadelphia, PA: Elsevier. 